\title{
INSCRIPCIONES DE GUADALAJARA, ÁVILA, ALCONÉTAR, SEGOBRIGA Y SEVILLA EN MANUSCRITOS DE LA REAL ACADEMIA DE LA HISTORIA
}

\author{
JUAN MANUEL ABASCAL PALAZÓN \\ Universidad de Alicante \\ ROSARIO CEBRIÁN FERNÁNDEZ \\ Parque Arqueológico de Segobriga
}

\begin{abstract}
Presentamos dos inscripciones inéditas de Guadalajara, algunas correcciones sobre la procedencia de otras y nuevas ilustraciones epigráficas de manuscritos de la Real Academia de la Historia (Madrid).

This text includes two new roman inscriptions from Guadalajara, many topographica modifications and new images for roman texts, all in manuscripts of the Royal History Academy (Madrid).
\end{abstract}

\section{DOS NUEVAS INSCRIPCIONES ROMANAS DE GUADALAJARA (FIGS. 1 Y 2) ${ }^{1}$}

El archivo de la Real Academia de la Historia alberga tres documentos relativos a dos inscripciones romanas de Guadalajara desconocidas hasta el presente.

El primero de ellos es una carta de Justo España y Romero, Archivero del Cabildo de Curas y Beneficiados de Guadalajara, a Diego Clemencín, fechada en Guadalajara el 10 de mayo de 1819 , comunicándole el descubrimiento de algunas monedas romanas y de dos inscripciones (RAH-11-8055-39a). Es un pliego en $4^{\circ}$ con cuatro páginas de texto donde se describen detalladamente las circunstancias de hallazgo y conservación de los objetos citados. El segundo documento (RAH-11-8055-39b) es otro pliego en $4^{\circ}$ fechado en Guadalajara el 9 de mayo de 1819 y contiene los dibujos realizados por el M(aest)ro Arquitecto Principal de las R(eale)s Fabricas [de Paños de Guadalajara] de las dos inscripciones citadas, dibujos a los que Justo España y Romero añadió algunos comentarios específicos sobre el hallazgo de ambos monumentos. El tercer documento (RAH-11-8055-39c) es una minuta de oficio firmada por Clemencín el 19 de mayo de 1819 , dando a Justo España las gracias por sus noticias de Guadalajara.

En su carta de 10 de mayo, Justo España indica que con frecuencia se encuentran en los alrededores de Guadalajara monedas anti- guas, de las cuales entregó algunas a José García de la Torre, quedando ya sólo una en su poder; se refiere también al hallazgo en el río Henares de dos inscripciones romanas y a la conservación en el archivo municipal de algunos documentos de interés.

En la hoja con los dibujos de las inscripciones, fechada el 9 de mayo de 1819 (RAH11-8055-39b), se explican con detalle las circunstancias del hallazgo, que reproducimos integramente:

Estas dos Piedras se hallaron $p(0) r$ los años de 1788 en el Zampeado del Puente de Guadalax(a)ra del Rio Nares y p(o)r mandado del Intend(en)te Correx(ido)r D(o)n Miguel de Vallejo se condujeron a la R(ea)l Fabrica donde se hallan.

Se debe tener pres(en)te $q(u)$ e habiendose acabado de reedificar dos ojos de $d($ ic) ho Puente en el año de 1776, el d(ic) ho Zampeado $q(u)$ e se hizo nuebo quedo limpio, y sin el mas ligero escombro. En el año de 1777 y siguientes, ocurrieron en el Rio grandes avenidas, $y$ tales $q(u)$ e nunca se habian visto, y luego aparecieron en el Zampeado. No es facil atinar de donde pudieron ser arrancadas hasta $d($ ic) ho sitio, pues hai ocasiones de alubiones con crecidos en Gua(dalaja)ra q(u)e desde la Montaña $p(o) r$ el foso oriental de la Ciudad forman un

\footnotetext{
${ }^{1}$ La redacción de este trabajo se ha realizado en el marco del proyecto BHA2003-04593 financiado por el Ministerio de Ciencia y Tecnología y fondos FEDER.
} 
grande Rio descendiendo con grande impetu al Nares y Puente y como en el foso se notan vestigios de murallas, y otros edificios ruinosos con piedras labradas y sin labrar, $q(u)$ e no es mui dificil arrancar, pudieron mui bien socabar las aguas $d(i c)$ has Piedras, $y$ despues llebarselas los alubiones hasta d(ic)ho Zampeado. Si esto es asi ambas Piedras fueron colocadas en Guadalaxara en t(iem)po de los Romanos, pero tambien pudieron ser trahidas $p(o) r$ las aguas del Nares en las $d($ ic) has grandes avenidas desde Humanes, en cuyas inmediaciones aseguran algunos estubo la antigua Arriaca negando $q(u)$ e Guad(alaja)ra tubiese aquel nombre, ni el de Caraca; pero no se puede negar q(u)e Guadalax(a)ra fue Poblacion considerable en $t$ (iem)pos de los Romanos, y Godos, $p(o) r q(u) e$ se conserban algunos edificios de ellos, y se hallan frequentemente monedas, $y$ otros vestigios $q(u)$ e lo comprueban.

El M(aest)ro Arquitecto principal de las $R$ (eale)s Fabricas me entregó esta demostración de las dos Piedras y me ha parecido remitirla original a la R(ea)l Academia. Gua(dalaja)ra, 9 de Mayo de 1819. Justo España y Romero (rubricado).

De la carta de Justo España se desprende que ambas inscripciones llegaron a los pilones del río Henares entre 1776 y 1788, fecha de su descubrimiento. Las explicaciones que proporciona para justificar la presencia de ambos monumentos, teniendo su lógica, no dejan de ser imaginativas; es verdad que el Barranco del Alamín, el que llama foso oriental es una rambla natural que ha conocido importantes crecidas, pero cuesta aceptar que dos piedras de esta envergadura -una de ellas una cupafueran arrastradas durante tanto trayecto. Más imposible aún es que procedan de la zona de Humanes, situada ya a varias decenas de kilómetros. Lo más lógico es suponer que en las inmediaciones del puente de Guadalajara sobre el río Henares hubiera alguna pequeña necrópolis cercana al río y que el agua socavara las márgenes hasta hacer que estas cayeran al cauce. Respecto al lugar de conservación, Real Fábrica de Guadalajara establecida en 1719 (González Enciso, 1975; 1980; Villaverde, 1981), no tenemos ninguna otra noticia sobre la conservación allí de las inscripciones ni de su paradero posterior.

Los dibujos y notas del M(aest)ro Arquitecto Principal de las $R$ (eale)s Fabricas, en donde se conservaban las inscripciones en 1819 , permiten hoy dar a conocer estos dos nuevos textos.
1. Funeraria (Fig. 1)

Esta Piedra es arenosa: Tiene de largo quatro pies y tres octabos: $y$ uno y tres quartos a la cabeza y dos pies al pie. Guarda la misma figura que se demuestra.

Estela en piedra arenisca de forma trapezoidal con una cartela cuadrada rehundida que alberga el texto y una línea superior en el marco. Sus dimensiones aproximadas eran $122,5 \mathrm{~cm}$ de altura $(1$ pie $=28 \mathrm{~cm}), 56 \mathrm{~cm}$ de anchura en el pie y $49 \mathrm{~cm}$ de anchura en la cabecera; no hay datos de su grosor. Hallada en 1788 en el Zampeado del Puente de Guadalax(a)ra del Rio Nares y trasladada a la Real Fabrica de Paños de la ciudad, donde se encontraba en 1819 (España, 1819, ms.). Perdida.

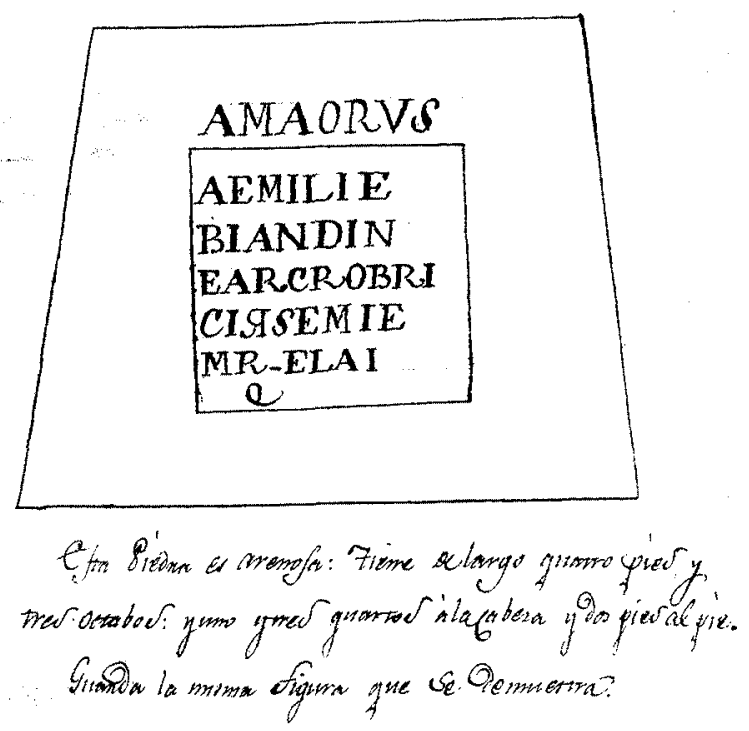

Figura 1: Inscripción n. ${ }^{\circ} 1$ de Guadalajara en el manuscrito RAH-11-8055-39b.

A primera vista, el texto recogido en el manuscrito es el de una inscripción funeraria. El primero de los nombres citados dentro de la cartela puede leerse sin dificultad; Aemilie Blandine no es más que una forma gráfica de Aemili(ae) Blandin(a)e, el dativo o genitivo del nombre de una difunta llamada Aemilia Blandina; nótese que el dibujante confundió la $L$ de Blandine con una I por tener seguramente el trazo horizontal corto, lo que debe ser tenido en cuenta en el resto de la inscripción. Tras este nombre parece encontrarse la origo de esta mujer: la cuarta línea termina con el texto ARCROBRI; al comienzo de la quinta se ve una $C$ ó $G$, seguida de un asta vertical y lo que se interpretó como una $\mathrm{R}$ en posición invertida; esta falsa $\mathrm{R}$ no es otra cosa que las letras $\mathrm{SI}$, que pudieron confundir por su proximidad al autor del dibujo, dándole la impresión de R; de este modo, en la I que las precede habría que 
suponer una $E$ mal leída por tener los trazos horizontales muy cortos. Con las debidas precauciones, podríamos deducir que entre las líneas 4 y 5 se encuentra la forma de dativo $\operatorname{Arc}\{r\}$ obrige(n)si, y menos probablemente el genitivo Arc\{r\}obrige $(n)$ sis, pues la $S$ final debería ser una mala lectura de la primera letra del siguiente nombre (vid. infra).

Entre las líneas 5 y 6 aparece otro nombre personal: en 6 es fácil ver el nombre Mar[c]cel(l)a, escrito originalmente con nexo MA y con una sola $L$, con la $C$ borrada por el deterioro del monumento. El nomen de esta mujer se encuentra abreviado al final de la línea 5: en el dibujo se lee SEMIE; con las debidas precauciones, y considerando la presencia de un nombre similar en la línea 2, podriamos pensar que la $S$ inicial fuera confundida con una $A$ de trazo horizontal oblicuo y que la $E$ final sea una $L$ que el deterioro de la pieza hizo confundir con L; de este modo, la opción más viable para el final de la línea 5 es AEMIL, es decir, Aemil(ia). No habría que descartar de entrada una forma como Sempr(onia), aunque parece difícil que el copista confundiera las letras PR con el grupo IE que recoge en su dibujo.

En todo caso, si en el nombre de la difunta Aemilia Blandina la desinencia AE se representa como $\mathrm{E}$, habría que descartar que en el nombre de Aemilia Marcella se escriba íntegramente la desinencia $\mathrm{AE}$; aunque ambos usos son corrientes, no es normal que aparezcan en un mismo texto. Por eso parece conveniente pensar que el nombre de la primera mujer está escrito en dativo y el de la segunda en nominativo. Ello lleva a ver en la I final de la línea 6 el asta izquierda de una letra con la que podrían introducirse fórmulas como $m$ (atri) o $f$ (iliae) más que una $E$ de la desinencia de dativo del nombre de la segunda mujer: En estas condiciones, Aemilia (?) Marcella parece ser la dedicante de la inscripción. Al final del texto, en la séptima línea, se encuentra lo que parece una $Q$, que debe ser una letra mal leída en una fórmula funeraria final.

Queda por establecer la lectura de la primera línea. Aquí cabría esperar una fórmula como D(is) Manibus, difícilmente encajable con el AMAORVS que aparece en el manuscrito, $y$ menos probablemente un dedicante en nominativo que parece encontrarse al final del texto, pues una opción como Amandus (Abascal, 1994,269 ), que sería la más ajustada, no parece encajar completamente con las letras dibujadas.
Con las debidas precauciones, del dibujo de este manuscrito se podría desprender la siguiente lectura:

\{AMAORVS\} D(is) Manibus (?)

Aemili(a)e

Blandin-

(a)e Arc\{r\}obri-

ge(n)si Aemil(ia)

Mar[c]el(l)a m[atri ?] Nexo MA (?)

[---] Q [---]

Los nombres citados en la inscripción son sobradamente populares en Hispania (Abascal, $1994,67, .412$ ) excepto Blandina (Abascal, 1994, 301), cognomen sólo conocido en la Península lbérica por dos testimonios de Singilia Barba $\left(C / L\left\|^{2} / 5,798=\right\| 2021\right)$ y Castulo $(A E$, 1958, 8).

ta ciudad de origen de Aemilia Blandina es Arcobriga ${ }^{2}$, situada tradicionalmente en el Cerro Villar de Monreal de Ariza (Zaragoza), en el trazado entre Segontia y Bilbilis de la vía Augusta Emerita - Caesaraugusta; pertenecía al conventus Caesaraugustanus y alcanzó el privilegio municipal en época flavia (Alföldy, 2001, 250).

El hecho de que Guadalajara se encuentre en el paso de esa importante arteria de comunicaciones que era la vía Augusta Emerita Caesaraugusta favoreció el desplazamiento hacia aquí de gentes procedentes de las ciudades situadas en ese recorrido. Conocíamos ya el epigrafe funerario del Segontinus $M$. Messius Abascantus en las cercanias de Azuqueca ( $A E 1987,635)$, al que se suma ahora el de esta Arcobrigensis.

\section{Funeraria (Fig. 2)}

Esta Piedra goza la figura á forma de cofre tambor y en su frente esta la inscripcion. Tiene dos pies de ancho, y dos pies y medio de alto.

Cupa en la que el texto ocupaba uno de los lados menores. Sus dimensiones eran $70 \mathrm{~cm}$ de altura y 56 de anchura, sin que tengamos datos de su profundidad. Hallada en 1788 en el Zampeado del Puente de Guadalax(a)ra del Rio Nares y trasladada a la Real Fabrica de Paños de la ciudad, donde se encontraba en 1819 (España, 1819, ms.). Perdida.

\footnotetext{
2 Plin., N.H. 3, 24; Ptol. II, 6, 57; Itin. Ant. 437, 1 y 438,13; Ravenn. 309,17. Sobre las ruinas de la ciudad, cf. Lostal, 1980, 200-207; Beltrán Lloris, 1988. La promoción de la ciudad y su adscripción a la tribus Quirina ha sido discutida en Alföldy, 2001, 239-250.
} 


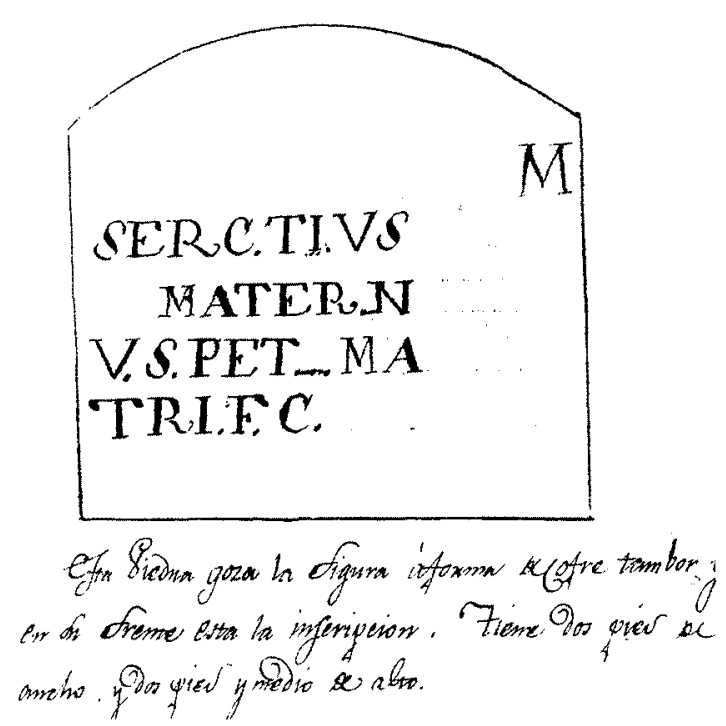

Figura 2: Inscripción n. ${ }^{\circ} 2$ de Guadalajara en el manuscrito $\mathrm{RAH}-11-8055-39 \mathrm{~b}$.

Este segundo monumento, en forma de cofre tambor como dice el dibujante, es una cupa de las que ya conocemos varios ejemplares en este tramo del valle del Henares. En la primera línea queda la segunda letra de la fórmula inicial, con lo que parece que en el texto decia $[D(i s)] M$ (anibus). Entre las lineas 2 y 4 se encuentra el nombre del dedicante en nominativo, cuyo cognomen Maternus parece claro. Para su nomen el dibujante recoge la forma SERCTIVS, que en buena lógica deberia ser una versión de Sergius, entorpecida por alguna fisura original o golpe accidental en el soporte que le indujera a dibujar una segunda asta vertical; menos probables son soluciones como Serotius, no atestiguada hasta ahora (cf. Solin y Salomies, 1988, 169: Serotinius). El dedicante sería casi homónimo del $M$. Sergius Maternus de Torredonjimeno (Jaén. C/L II/2/5, 59).

En la línea 4 habría que esperar el nombre de la difunta en dativo a juzgar por la forma MATRI que aparece a continuación. Sin embargo, el escaso espacio que deja ver el dibujo y la breve relación de cognomina latinos que encajan en esa secuencia (Solin y Salomies, 1988, 378; Abascal, 1994, 455) hacen imposible cualquier propuesta; tampoco hay que descartar que el nombre de la madre aparezca indicado por su nomen en dativo, con lo que serian factibles soluciones como Pet[eliae], Pet[iciae], Pet[iliae], Pet[reiae] o incluso Pet[roniae], en todo caso en una laguna más grande que la que ofrece el dibujo.

De lo dicho, se desprende que el texto debía decir lo siguiente:
[D(is)] M(anibus)

Serg\{t\}ius

Matern-

us Pet[---] ma-

tri f(aciendum) $c$ (uravit)

El uso de las cupae como monumento funerario con inscripción está muy bien documentado en Hispania, donde ha ido creciendo en los últimos años el número de ejemplos por diferentes regiones (Abascal, 1995, 75-76; Abascal y Ramallo, 1997, 33-34). En la más cercana a Guadalajara, en el territorio de la antigua Complutum, Stylow ha llegado a documentar hasta ocho monumentos de esta forma, alguno tan cercano a este nuevo testimonio como el de Alovera ( $A E$ 1999, 932 y las mejoras de lectura hechas ahora por Stylow), lo que parece indicar que, efectivamente, los epígrafes funerarios en forma de cupa gozaron de una cierta popularidad en esta comarca.

\section{LAS INSCRIPCIONES DE LA MURALLA DE ÁVILA (FIGS. 3 Y 4)}

El legajo 21 de la Colección Villanueva de la Real Academia de la Historia (RAH-94578; olim Est 19, gr $4^{\mathrm{a}}, \mathrm{n}^{\circ}{ }^{\circ}$ 81) contiene un conjunto de notas, casi todas de epigrafía y numismática, reunidas por Joaquín Lorenzo Villanueva (Xátiva 1757 - Londres, 26 de marzo de 1837), tanto por sí mismo como a través de correspondientes, en los primeros años del siglo XIX; el legajo aparentemente pasó desapercibido para Hübner y sus colaboradores pese a que, como hemos podido comprobar, tiene un gran interés para el estudio de las inscripciones hispano-romanas. Destaca en él la serie de los datos sobre inscripciones de los territoria de Saetabis y Edeta (Abascal y Cebrián, 2004) ${ }^{3}$, el catálogo de las inscripciones expuestas en el Ayuntamiento de Cartagena (Murcia) en 1810 y nunca hasta ahora identificadas (RAH-9-7584-1 y 9; Abascal y Cebrián, 2005), algunas notas sobre epígrafes ya conocidos de Cataluña y una hoja sobre inscripciones de Ávila de la que nos ocupamos aquí (RAH-9-4578-5).

La hoja en cuestión es un pequeño papel en $8^{\circ}$, de tan sólo $16 \times 11 \mathrm{~cm}$, con manchas en su ángulo superior izquierdo y escrita por las dos caras. Aunque no está fechada, la letra parece propia del siglo XVII y en el extremo

\footnotetext{
${ }^{3}$ RAH-9-4578-2a-c; 9-4578-4; 9-4578-6; 9-4578-12; 9-4578-13
} 


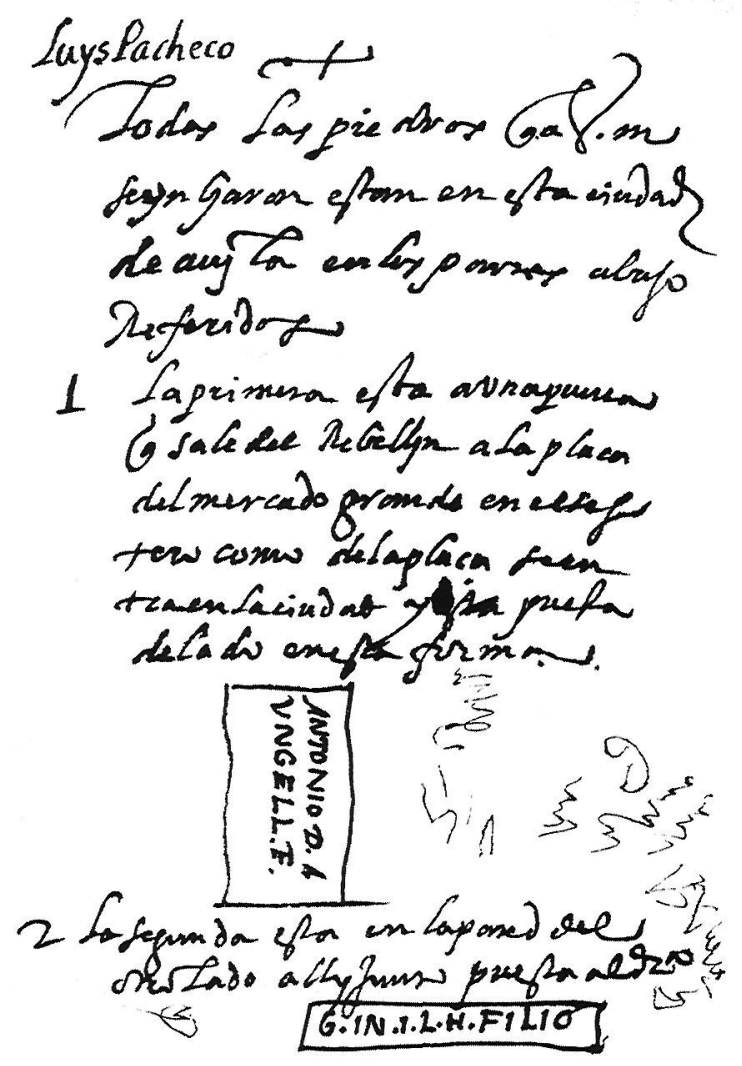

Figura 3: Manuscrito RAH-9-4578-5. Cara anterior.

superior izquierdo, con una caligrafía distinta y claramente dieciochesca, se lee Luys Pacheco, lo que permite adjudicar estas notas a Luis Pacheco de Espinosa, autor de una Historia de Ávila en 1607 que conserva la Academia (RAH9-4667). Hay que imaginar que Joaquín Lorenzo Villanueva o quien le proporcionó este papel trataron de identificar con este breve apunte al autor de las notas, en una época en que aquello aún era posible.

Este breve autógrafo de Luis Pacheco contiene la trascripción de cuatro inscripciones empotradas en la muralla de Ávila y al final alude a una quinta que figuraba en una segunda hoja hoy perdida. Tras una primera lectura del texto pensamos que se trataba de una copia obtenida de la Historia de las grandezas de la ciudad de Ávila de Luiz Ariz (Ariz, 1607/1978; cf. Knapp, 1992, 313-316), pues dos de los textos ya aparecen en su obra, pero esta hipótesis queda descartada a la vista de la diferente ordinatio de ambas transcripciones, que en el caso del nuevo documento tampoco respeta los cortes de las líneas. La paleografía sugiere una redacción casi contemporánea a la de Ariz.

El texto del breve documento es el siguiente:

fol. $1 \mathrm{r}$ : :

Todas las piedras q(ue) a V.m. se ynbiaron estan

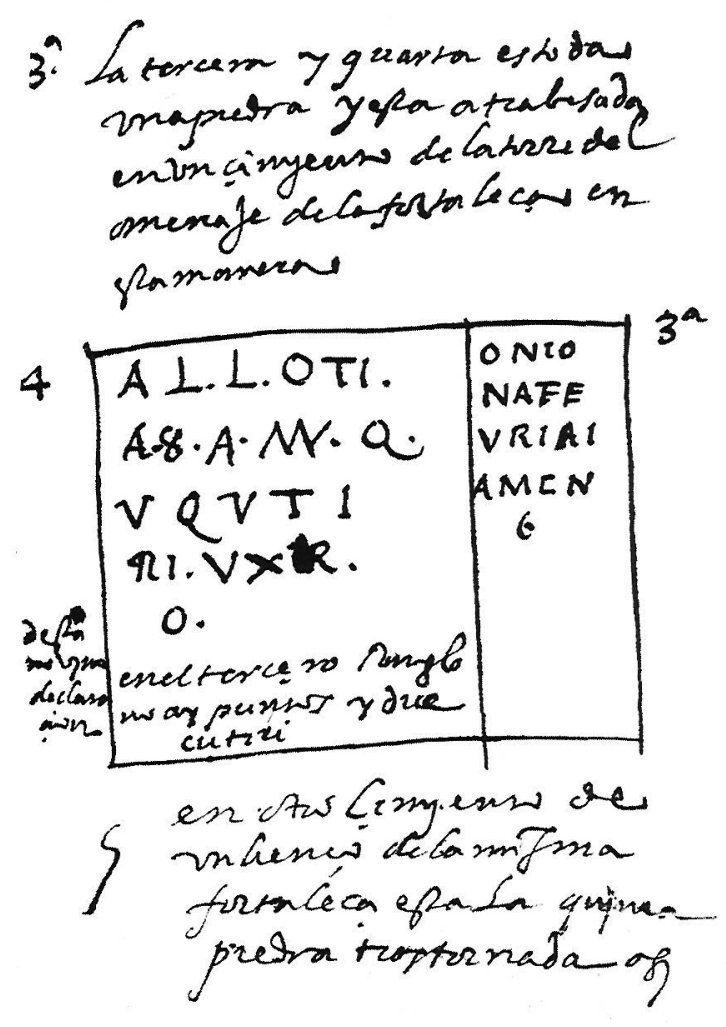

Figura 4: Manuscrito RAH-9-4578-5. Cara posterior.

en esta ciudad de Avyla con los porm(eno)res abajo referidos.

1. La primera esta a una puerta q(ue) sale del Rebellin a la plaça del mercado grande en el sentido como de la plaça se entra en la ciudad y esta puesta de lado en esta forma.

2. La segunda esta en la pared del su lado ally junto puesta al d(e)r(ech)o.

fol. $1 \mathrm{v}$.:

3. La tercera y quarta es toda una piedra y esta atrabesada en un cimyento de la torre del omenaje de la fortaleça en esta manera. En el tercero renglon no ay puntos y dice cutiri. Al margen: de esta no huvo declaracion.

$Y$ en otro cimiento de un lienço de la misma fortaleça esta la quinta piedra trastornada asi (vacat).

1. Se trata de CIL II 5865 (Knapp, 1992, n. ${ }^{\circ} 22$, actualmente en la torre 15 de la muraIla), de la que Hübner sólo conoció como edición antiguna la de Ariz. Como puede verse, el manuscrito de comienzos del siglo XVII no respeta ni siquiera la división de líneas del original.

2. Esta segunda inscripción de la torre 15 de la muralla abulense no fue conocida en la bibliografía hasta 1980 (editio princeps: Rodríguez Almeida, 1981, 116, n. 24; cf. Knapp, 1992, n. $\left.{ }^{\circ} 55\right)$. La lectura del manuscri- 
to es netamente peor que las lecturas posteriores pero se trata de la noticia más antigua que tenemos del monumento.

3. La inscripción n. ${ }^{\circ} 3$ del manuscrito de Luis Pacheco es CIL II 5869 (Knapp, 1992, n. ${ }^{\circ}$ 33 ), de la que Hübner sólo conoció como edición antigua la de Ariz. Curiosamente, la disposición real del monumento contiene en la parte izquierda el texto que Pacheco sitúa por error a la derecha; a cambio, en esta inscripción parece que el autor del manuscrito respetó la ordinatio del original.

4. La inscripción $n .^{\circ} 4$ fue publicada por primera vez a finales del siglo XIX y en el catálogo de Knapp (1982, n. $\left.{ }^{\circ} 17\right)$ aparece como descubierta en 1901. Ahora sabemos por esta scheda de la Real Academia de la Historia, que nada aporta a su lectura correcta, que se conocía desde comienzos del siglo XVII.

El manuscrito de Pacheco señala erróneamente que esta inscripción $\left(n .^{\circ} 4\right)$ y $\mathrm{CIL}$ II 5869 ( $n .^{\circ} 3$ ) estaban grabadas en un mismo bloque empotrado en la Torre del Homenaje. Sin embargo, en la piedra que contiene en su parte izquierda el texto CIL II 5869, la parte derecha del bloque no presenta restos de inscripción, ni siquiera débiles trazas de su antigua existencia; por la disposición de lo conservado, que parece responder a una división del soporte para dos cartelas, no se puede descartar que en su origen hubiera aquí algún texto, pero esto es hoy imposible de determinar. En todo caso podemos rechazar abiertamente que CIL II 5869 (Pacheco, n. ${ }^{\circ} 3$ ) y Knapp 1982, n. ${ }^{\circ}$ 17 (Pacheco, n. ${ }^{\circ} 4$ ) estuvieran grabadas en una misma piedra.

Resulta curiosa, sin embargo, la noticia dada por el autor de comienzos del siglo XVII sobre la proximidad en la muralla de ambos epígrafes. Aun admitiendo su error al considerarlos parte de un mismo monumento, podría pensarse que en esas fechas se encontraban tan próximos como para inducir a la confusión. CIL II 5869 aparece citada siempre en el mismo emplazamiento que da Pacheco (n. ${ }^{\circ} 3$ ), es decir, en la Torre del Homenaje; el texto Knapp 1982, n. ${ }^{\circ} 17$ (Pacheco n. ${ }^{\circ} 4$ ) se encontraba en 1901 en la que Knapp Ilama torre 18, contigua a la anterior. Esta relativa proximidad podría explicar la confusión de Pacheco.

\section{LOS ULENSES DE ALCONÉTAR (GARRO- VILLAS, CÁCERES) (FIG. 5)}

Desde el siglo XIX se hace proceder de la muralla del castillo de Uclés (Cuenca), en territorio de Segobriga, un altar de granito propiedad de la Real Academia de la Historia ${ }^{4}$ que hoy guarda el Museo de Segobriga. Según Pelayo Quintero $(1913,109)$ fue retirado por Román García Soria de la citada muralla de Uclés y donado a la Academia, circunstancias que nadie ha podido rebatir pese a que diversos elementos hacían sospechar de la procedencia establecida.

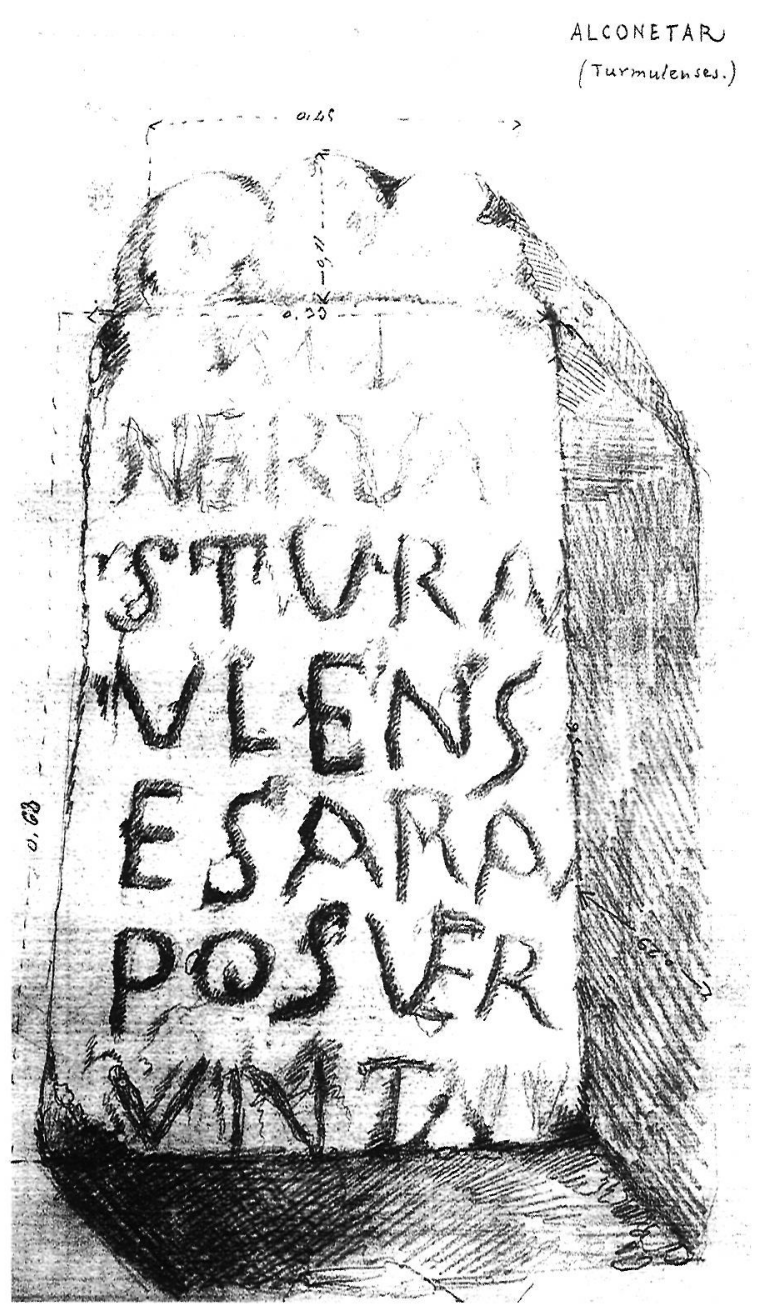

Figura 5: Inscripción de Alconétar en el manuscrito RAH-97363-78.

Las dudas derivaban en primer lugar del material del soporte, pues se trata del único monumento de granito dentro del conjunto

\footnotetext{
4 Hübner, CIL II 5877, que la vio en la Academia (= Vives, ILER 738 y 1008); Rivero, 1933, n. ${ }^{\circ} 21$; Almagro Basch, 1984, $80-82, n .{ }^{\circ} 14$, con el resto de la bibliografía; Abascal y Gimeno, 2000, 116-117, n. ${ }^{\circ} 150$. Junto con otras piezas del Gabinete de Antigüedades de la Real Academia de la Historia, fue depositada en el Museo Arqueológico Nacional el 28 de junio de 1907 (exp. 1907/32/52; inv. n. ${ }^{\circ} 38.394$ ); el 10 de enero de 1974 fue trasladada al Museo de Segobriga, donde hoy se encuentra.
} 
epigráfico conservado en Segobriga; en segundo lugar, de ser cierta la lectura habitual de la primera línea, sería ésta la única dedicación a Ataecina aparecida fuera del contexto del suroeste de la Península Ibérica si exceptuamos un epígrafe de Cerdeña. En tales circunstancias ya M. Almagro-Gorbea supuso que la inscripción debía proceder de algún lugar más próximo al área extremeña (Almagro-Gorbea, 1995, 91, nota 3; Abascal, 1995, 91, notas 256257 ), en contra de lo cual sólo militaba la noticia de Hübner que la vio en la Academia con un rótulo indicando la procedencia de $\mathrm{Se}$ gobriga, argumento que fue aceptado por Fidel Fita y Juan de Dios de la Rada $(1889,141)$.

Las dudas parecen definitivamente resueltas gracias a un dibujo anónimo de esta inscripción que hemos encontrado en el Archivo de la Real Academia de la Historia (RAH-97363-78). Se trata de un dibujo a carboncillo $(31,5 \times 21 \mathrm{~cm})$ en cuyo ángulo superior derecho se lee con toda claridad: «ALCONETAR (Turmulenses)»; esta anotación devuelve la inscripción al área extremeña de culto de Ataecina y soluciona las dudas planteadas por la presencia del granito en el conjunto de Segobriga.

\section{ALTAR DE LEIOSSA DE SEGOBRIGA (FIG. 6)}

CIL II 3097 + p. 944 (= ILER 863); Rada y Fita, 1889, 127 y 141; Blázquez, 1962, 213; Almagro Basch, 1984, 86-87, n. ${ }^{\circ} 17$.

Cuando Hübner publicó esta inscripción en CIL II 3097 fue avisado por Pascual de Gayangos de que existía un dibujo del monumento en la Academia (Supra ipsum lapidem delineatam dedit Gayangos); la información se concretó en CIL II supp. cuando Fita ya había visto el dibujo y habia adjudicado su autoria a Juan Antonio Fernández (CIL II supp., p. 944: In ectypo, quod servat Gayangos, Antonio Fernandez adscripsit ... quod mihi indicavit Fita).

El dibujo en cuestión forma parte de lo que hemos denominado el Diario de excavaciones de Juan Antonio Fernández y el obispo Antonio Tavira, que contiene los datos de sus trabajos en Segobriga entre octubre de 1789 y enero de 1790 (RAH-11-8109-4k). Se trata de un conjunto de pliegos y hojas sueltas escritas de mano de Fernández, muy deteriorado y originalmente cosido en un solo volumen, que contiene apuntes, croquis, diferentes versiones de un mismo dibujo, anotaciones con indicación de hallazgos en fechas concretas, etc. Se trata del cuaderno de campo del autor durante el período de excavaciones, desencuadernado y posteriormente vuelto a encuadernar sin orden cronológico.

En la página $17 r$ de este volumen se encuentra un papel doblado $(39 \times 21 \mathrm{~cm})$ con el dibujo a tamaño real del altar CIL II 3097. Al dorso aparecen algunas anotaciones para su estudio y una señal para indicar que su profundidad era de $14 \mathrm{~cm}$. El interés de este dibujo se acrecienta por haberse perdido el monumento y no quedar de él ninguna otra imagen.

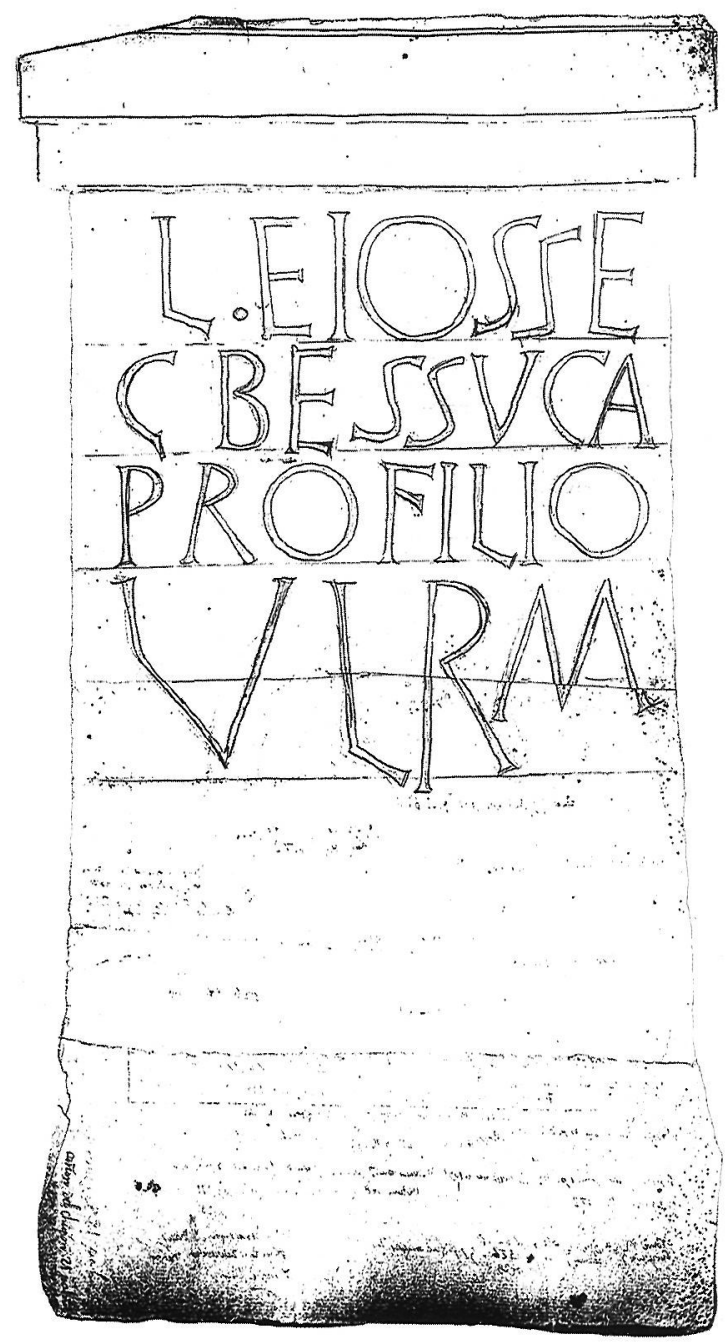

Figura 6: Altar de Segobriga en el manuscrito RAH-11-8109-4k.

Se trata de un altar con zócalo y coronamiento, formado este último por dos fajas rectas y sin pulvini. El fuste mide $26 \times 18 \mathrm{~cm}$ y contiene cuatro líneas de texto que ocupan sólo la parte superior de la superficie disponible, dejando por abajo un espacio libre de $8,5 \mathrm{~cm}$ de altura. Presenta líneas horizontales de pautado y las letras parecen bien grabadas aun- 
que con ligeras diferencias de tamaño entre ellas.

Las dimensiones completas del altar, hoy perdido, eran de $39 \times 20,5 \times 14 \mathrm{~cm}$. La altura de las letras es de $4,3,3$ y $5,5 \mathrm{~cm}$ en las cuatro líneas del texto, aunque en la última hay importante diferencias y la R llega a los $6,5 \mathrm{~cm}$. Apareció entre octubre de 1789 y enero de 1790 en las excavaciones en Segobriga. Perdida. El texto decía:

Leioss(a)e

C(---) Bessuca

pro filio

$v$ (otum) I(ibens) r(---) m(erito)

El sentido del texto es claro en el dibujo. En la propuesta de Hübner para desarrollar las abreviaturas del texto se lee C(ornelia?) Bessuca ... r(eddidit). A la vista del registro onomástico de Segobriga sería quizá más conveniente suponer que el nombre de la mujer fuese Caecilia, cuyo cognomen Bessuca sigue siendo hoy un unicum Respecto a la fórmula final no hay alternativas más convincentes que la de CIL II.

Lo verdaderamente importante es disponer ahora de una primera imagen del monumento y poder confirmar la lectura que de él se hizo hace más de cien años, incluyendo el mantenimiento del teónimo. En la primera línea, deberíamos suponer la presencia de una divinidad indígena en dativo bajo la forma Leioss(a)e.

\section{DOS INSCRIPCIONES CRISTIANAS DE LA PROVINCIA DE SEVILLA (FIGS. 7-9)}

1. IHC 60. Alcalá del Río (Sevilla). Ermita de San Gregorio (Figs. 7-8)

A la documentación existente deben añadirse dos dibujos realizados por Francisco Javier Delgado a finales de 1821 (RAH-9-736376).

Al pie del primero de los dibujos dice: Marmol q(u)e al parecer es una losa de marmol blanco empotrado en uno de los lados del altar y sepulcro de S(an) Gregorio llamado Osetario en la hermita de la V(ill)a de Alcalá del Río: de alto cinco palmos eseasos menos dos pulgadas, su ancho media vara, los caracteres, pulgada y media, y el circulo del monograma de. siete pulgadas poco mas ó menos: La copié $p(0) r$ el original à fines de 1821 , los numeros de la Era deben ser así: LXXXII esto es las $X$

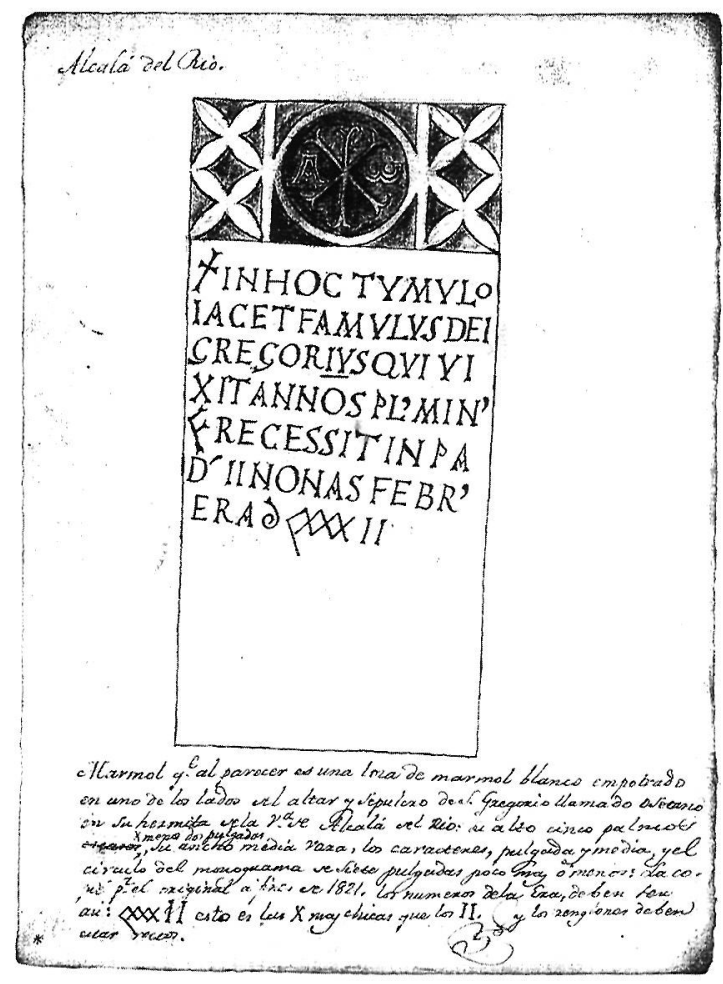

Figura 7: Manuscrito RAH-9-7363-76. Cara anterior.

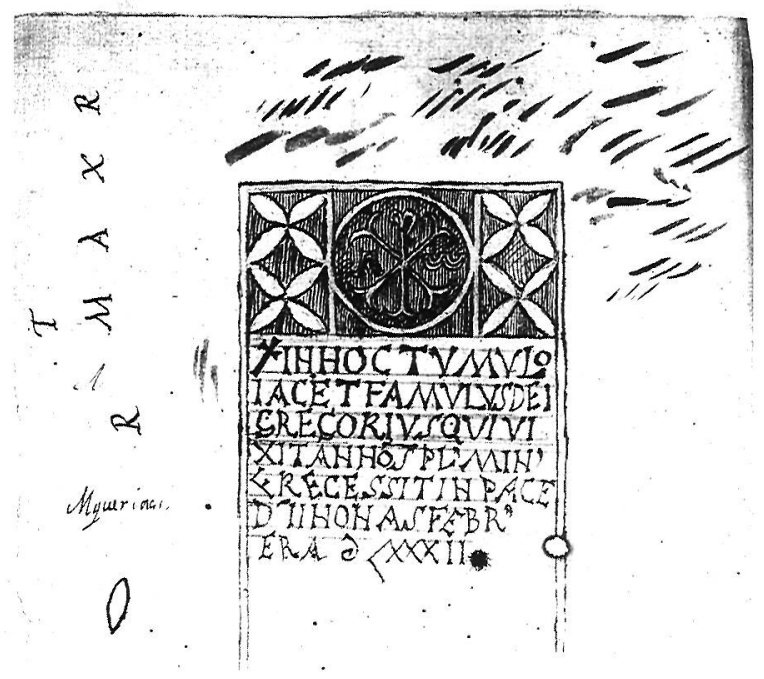

Figura 8: Manuscrito RAH-9-7363-76. Cara posterior.

mas chicas que los $1 /$ y los renglones deben estar rectos [sigue la rúbrica de Delgado].

\section{IHC 70. Sevilla (Fig. 9)}

A la documentación existente debe añadirse el dibujo y las notas tomadas por Francisco Javier Delgado en 1823 (RAH-9-7363-77). Hübner ( $a d . J H C$.70) aclara la identidad del anónimo autor del dibujo y del comentario: Francisc. Xav. Delgado descripsit a. 1823; habui ex schedis eius servatis apud Antonium filium; 
una de esas schedae es ésta que se conserva en la Real Academia de la Historia.

$\forall i$

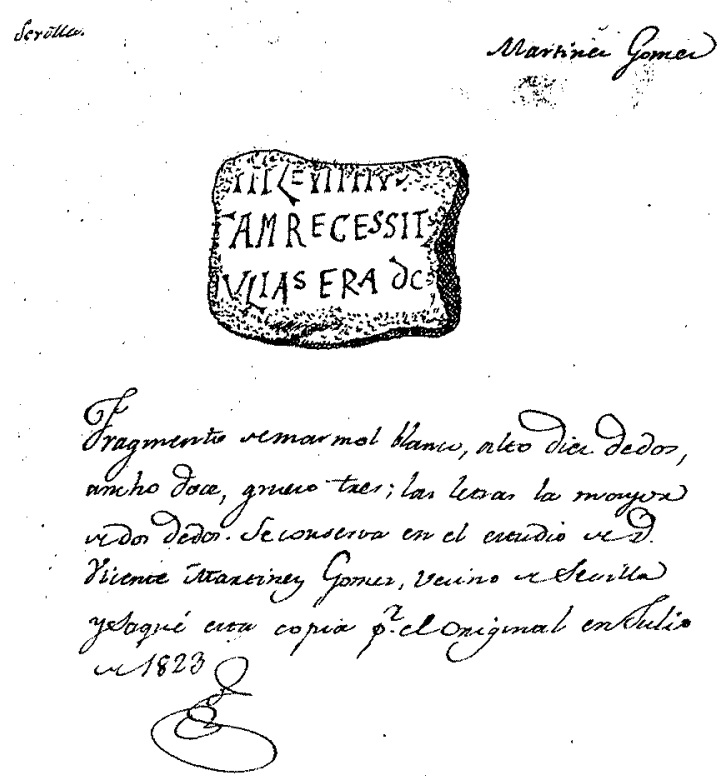

Figura 9: Manuscrito RAH-9-7363-77. Cara anterior.

En la ficha de Francisco Javier Delgado se dice: Fragmento de marmol blanco, alto diez dedos, ancho doce, grueso tres; las letras la mayor de dos dedos. Se conserva en el estudio de $D$ (on) Vicente Martinez Gomez, vecino de Sevilla y saqué esta copia p(o)r el original en Julio de 1823 [sigue la rúbrica de Delgado].

Una vez más se pone de manifiesto que tan importante como la recuperación de nuevos epígrafes es la búsqueda de la documentación antigua en archivos, tarea imprescindible en la que todos los esfuerzos son pocos.

Prof. J. Manuel Abascal Palazón Area de Historia Antigua Dpto. de Prehistoria, Arqueología, $\mathrm{H}^{\mathrm{a}}$ Antigua, Filología Griega y

Filología Latina

Facultad de Filosofía y Letras

Universidad de Alicante

Apdo. 99

03080 Alicante

juan.abascal@ua.es
ABASCAL, J. M. y CEBRIÁN, R., 2004: «Los informantes valencianos de Jaime Villanueva y las inscripciones romanas de los territorios de Edeta y Saetabis (Hispania Citerior)", Archivo de Prehistoria Levantina, 25, 345-357.

ABASCAL, J. M. y CEBRIÁN, R., 2005: La epigrafía y la arqueologia de Cartagena en la Real Academia de la Historia, Murcia.

ABASCAL, J. M. y GIMENO, H., 2000: Epigrafia Hispánica. Real Academia de la Historia. Catálogos del Gabinete de Antigüedades, Madrid.

ABASCAL, J. M. y RAMALLO, S. F., 1997: La ciudad de Carthago Nova III. La documentación epigráfica, Murcia.

ALFÖLDY, G., 2001: «Arcobriga in Hispanien, ein flavisches Municipium», ZPE, 136, 2001, 239-250

ALMAGRO BASCH, M., 1984: Segobriga II. Inscripciones ibéricas, latinas paganas y latinas cristianas, Excavaciones Arqueológicas en España 127, Madrid.

ALMAGRO-GORBEA, M., 1995: «El Lucus Dianae con inscripciones rupestres de Segobriga», en A. Rodríguez Colmenero, y L. Gasperini (eds.): Saxa Scripta (inscripciones en roca). Actas del Simposio Internacional IberoItálico sobre epigrafia rupestre. Santiago de Compostela y Norte de Portugal, 29 de junio a 4 de julio de 1992, Anejos de Larouco 2, 61-96, Coruña.

ARIZ, L., 1607/1978: Historia de las grandezas de la ciudad de Ávila. Alcalá de Henares; reedición de 1978.

BELTRÁN LLORIS, M., (dir.) 1988: Arcobriga (Monreal de Ariza, Zaragoza), Zaragoza.

BLÁZQUEZ, J. M. ${ }^{a}, 1962$ : Religiones primitivas de Hispania. Fuentes literarias y epigráficas, Madrid.

ESPAÑA, J., 1819, ms.: Carta de Justo España Romero a Diego Clemencín con los dibujos de dos inscripciones romanas de Guadalajara. Ms. RAH-11-8055-39b.

GONZÁLEZ ENCISO, A., 1975: «Inversión pública e industria textil en el siglo XVIII. La Real Fábrica de Guadalajara», Moneda y Crédito, 133, 41-64.

GONZÁLEZ ENCISO, A., 1980: Estado e Industria en el s. XVIII La fábrica de Guadalajara, Madrid.

KNAPP, R. C., 1992: Latin Inscriptions from Central Spain, Berkeley.

LOSTAL, J., 1980: Arqueologia del Aragón romano, Zaragoza.

QUINTERO, P., 1913: Uclés. Excavaciones efectuadas en distintas épocas y noticia de algunas antigüedades, Cádiz.

RADA, J. de Dios de la y FITA, F., 1889: «Excursión epigráfica a las ruinas de Cabeza de Griego», $B R A H, 15,1889,107-$ 151.

RIVERO, C. M. ${ }^{a}$ del, 1933: El lapidario del Museo Arqueológico Nacional., Catálogo ilustrado de las inscripciones latinas, Madrid.

RODRÍGUEZ ALMEIDA, E., 1981: Ávila romana, Ávila.

SOLIN, H. y SALOMIES, O., 1988: Repertorium nominum gentilium et cognominum Latinorum, Hildesheim-ZürichNew York.

VILLAVERDE, M. ${ }^{a}$ D., 1981: "La Real Fábrica de Paños y la ciudad de Guadalajara", Wad-al-Hayara, 8, 453-468.

\section{BIBLIOGRAFÍA}

ABASCAL, J. M., 1994: Los nombres personales en las inscripciones latinas de Hispania, Madrid.

ABASCAL, J. M., 1995: «Las inscripciones latinas de Santa Lucía del Trampal (Alcuéscar, Cáceres) y el culto de Ataecina en Hispania», Archivo Español de Arqueología, 68, 31-105. 